\title{
Isomer-specific effects of conjugated linoleic acid on blood pressure, adipocyte size and function
}

\author{
V. DeClercq ${ }^{1,2,3}$, C. G. Taylor ${ }^{1,2,4}$ and P. Zahradka ${ }^{1,2,4 *}$ \\ ${ }^{1}$ Department of Human Nutritional Sciences, University of Manitoba, Winnipeg, MB, Canada \\ ${ }^{2}$ Canadian Centre for Agri-food Research in Health and Medicine, Saint Boniface Hospital Research Centre, 351 Tache \\ Avenue, Winnipeg, MB, Canada R2H $2 A 6$ \\ ${ }^{3}$ Department of Biochemistry and Molecular Biology, Dalhousie University, Halifax, NS, Canada \\ ${ }^{4}$ Department of Physiology, University of Manitoba, Winnipeg, MB, Canada
}

(Submitted 2 March 2011 - Final revision received 15 June 2011 - Accepted 2 July 2011 - First published online 23 September 2011)

\section{Abstract}

Obesity-related hypertension may be caused by activation of the local adipose tissue renin-angiotensin system, resulting in exaggerated production of the vasoconstrictor angiotensin II. Additionally, secretion of adiponectin from adipose tissue, which prevents endothelial dysfunction, is altered in obesity. Consumption of conjugated linoleic acid (CLA) has been shown to modulate cytokine release from adipocytes and positively influence blood pressure in younger rats, but its physiological actions in older models with established hypertension and isomer-specific effects on adipocyte size remain to be determined. Therefore, we investigated the effects of CLA isomers on adipocyte size in relation to blood pressure and adipokine production by hypertrophic adipocytes in older $f a / f a$ Zucker rats with established hypertension. $f a / f a$ Zucker rats were fed with $\operatorname{cis}(c) 9$, $\operatorname{trans}(t) 11$-CLA or $t 10, c 12$-CLA isomers for 8 weeks and compared with lean and obese rats fed with the control diet. Blood pressure and adipocyte size were subsequently measured. Collagenase-isolated adipocytes were sizeseparated and angiotensinogen and adiponectin protein levels quantified by Western blotting. The t10,c12-CLA group had reduced blood pressure, fewer large adipocytes and increased serum adiponectin. Angiotensinogen was present at higher levels in the large adipocytes, whereas the converse was observed for adiponectin. The beneficial effects of the $t 10, c 12$-CLA isomer on blood pressure and adipocyte size in vivo may be due to its ability to reduce the number of large adipocytes, which alters the levels of vasoactive molecules secreted from adipose tissue.

\section{Key words: Adipokines: Adipose tissue: Conjugated linoleic acid: Hypertension}

The potential of conjugated linoleic acid (CLA) to prevent obesity-related diseases has gained much attention over the last several years. However, there are several gaps in our understanding of the effects of CLA on blood pressure regulation, especially in older animals with established hypertension, in relation to isomer-specific responses and their possible mechanisms of action. The rising incidence of obesity-related CVD such as hypertension has been linked, in part, to changes that affect the normal function of adipose tissue.

Normally, adipose tissue produces and secretes a range of bioactive molecules involved in the regulation of glucose and lipid metabolism, inflammation, blood pressure, coagulation and appetite control ${ }^{(1,2)}$. In obesity, growth of adipose tissue is initially due to enlargement of adipocyte size (hypertrophy), due to the increased delivery of fatty acids to adipose tissue, followed by an increase in adipocyte number

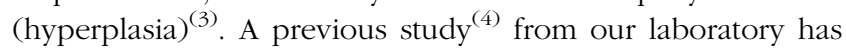
shown that feeding $f a / f a$ Zucker rats a mixture of CLA isomers has no effect on total body weight, but it does result in reduced epididymal adipocyte size. CLA isomers are naturally occurring fatty acids found in ruminant animals, with the most commonly studied isomers being the $\operatorname{trans}(t) 10, \operatorname{cis}(c) 12$ and the $c 9, t 11$-CLA isomers. We have recently shown that it is the $t 10, c 12$-CLA isomer that is responsible for the reduction in adipocyte size $\mathrm{s}^{(5)}$.

Previous study ${ }^{(6)}$ has shown that enlarged adipocytes have altered expression and secretion of pro- and anti-inflammatory adipokines, with large adipocytes preferentially displaying a pro-inflammatory state. Altered production and secretion of adipokines, such as adiponectin and angiotensinogen, from enlarged adipocytes may affect cellular processes associated with obesity-related hypertension ${ }^{(7)}$. In obesity, increased adipose tissue renin-angiotensin system activity enhances production of angiotensinogen and angiotensin II (AngII), resulting in elevated blood pressure ${ }^{(8-10)}$. Interestingly, the $t 10, c 12-C L A$ isomer of CLA has shown beneficial effects on

Abbreviations: AngII, angiotensin II; c, cis; CLA, conjugated linoleic acid; $t$, trans.

*Corresponding author: P. Zahradka, fax +12042374018, email peterz@sbrc.ca 
blood pressure in younger rats as well as a reduction in angiotensinogen mRNA in adipose tissue ${ }^{(11-13)}$. However, it is unknown if the $t 10, c 12$-CLA isomer has beneficial effects in older rats with established hypertension or affects production of angiotensinogen in enlarged adipocytes.

In contrast to angiotensinogen, adiponectin is reduced with increased adipose tissue accumulation ${ }^{(14,15)}$, and low levels have been associated with the development of obesity-related hypertension $^{(16)}$. Treatment with a CLA mixture also increased serum adiponectin and adiponectin mRNA levels in adipose tissue $^{(4)}$. Adiponectin is a $30 \mathrm{kDa}$ adipokine synthesised in adipose tissue. Before being secreted from adipose tissue, adiponectin undergoes post-translational modifications, allowing the protein to assemble into different oligomers ${ }^{(17,18)}$. Circulating adiponectin can be found in its trimeric, hexameric and high molecular weight forms ${ }^{(19)}$. Adiponectin exerts its physiological effects in target tissues by activating adenosine monophosphate-dependent protein kinase (AMPK) and PPAR through binding to adiponectin receptors (AdipoR1 and AdipoR2) ${ }^{(20)}$.

Although it appears that reducing cell size may beneficially influence the production and secretion of the blood pressureregulating adipokines, adiponectin and angiotensinogen, direct influence of production/secretion from hypertrophic adipocytes has not been adequately explored. Furthermore, it remains to be determined whether CLA isomers differentially regulate adipokine production in hypertrophic adipocytes, characteristic of obesity. Thus, we elected to investigate the effects of CLA isomers on adipocyte dysfunction in relation to blood pressure and adipokine production by large and small adipocytes in older $f a / f a$ Zucker rats with established hypertension. Many adipokines have been linked to blood pressure regulation ${ }^{(21)}$; however, the strongest links have been made with angiotensinogen ${ }^{(22,23)}$, adiponectin ${ }^{(16,24)}$ and leptin ${ }^{(25,26)}$. However, the animal model used in the present study has a mutation in the leptin receptor and thus would not respond to increases in leptin. Therefore, the present study focused on adiponectin and angiotensinogen as blood pressure-regulating adipokines.

\section{Experimental methods}

\section{Animals and diet}

The protocol used for these experiments was approved by the University of Manitoba Protocol Management and Review Committee. Male $\mathrm{fa} / \mathrm{fa}$ Zucker rats (15 weeks old) and their lean littermates (Harlan, Indianapolis, IN, USA) were randomly assigned to one of the three dietary groups ( $n 10$ per group): (i) obese control; (ii) $0.4 \%$ (w/w) c9,t11-CLA; (iii) $0 \cdot 4 \%(\mathrm{w} / \mathrm{w}) t 10, c 12-C L A ;$ (iv) lean rats fed with the same diet as the obese control group. The diet formulation has been previously published ${ }^{(27)}$. At the end of the 8 -week feeding period, the rats were euthanised by $\mathrm{CO}_{2}$ asphyxiation followed by decapitation. Blood samples were collected from the trunk of the animals, centrifuged at $3000 \boldsymbol{g}$ for $15 \mathrm{~min}$ at $4^{\circ} \mathrm{C}$ and subsequently stored at $-80^{\circ} \mathrm{C}$ until analysed for serum adiponectin using a rat-specific total adiponectin EIA kit (Alpco, Salem, NH, USA).

\section{Blood pressure measurements}

The indirect tail-cuff method (IITC Life Sciences blood pressure monitoring system, Woodland Hills, CA, USA) was used to measure systolic blood pressure in the conscious state at baseline and at the end of the present study. The tail-cuff method has been used for many years, and comparative studies show that both telemetry and the tail-cuff methods provide similar blood pressure results ${ }^{(28,29)}$. Three measurements were taken for each animal at each time point and the average was calculated to determine mean systolic pressure.

\section{Morphometry}

Epididymal adipose tissue samples frozen in cryogel were sectioned (10- $\mu \mathrm{m}$ thick), mounted on Super Frost plus slides (Fisher, Ottawa, ON, Canada) and fixed in formaldehyde. Images were captured with a BH2-RFCA microscope (Olympus, Center Valley, CA, USA) using a Q-Imaging camera and Q-Capture Pro 6.0 software (QImaging, Surrey, BC, Canada). Adipocyte size was quantified with Image J software 1.42 (National Institutes of Health, Bethesda, MD, USA), as previously described $^{(4)}$. A continuous block of twenty-five cells was measured in every field to determine average cell size and size distribution for each treatment group. In addition, the number of adipocytes in each $160 \mathrm{~mm}^{2}$ section was counted and the mean number of cells per group was quantified.

\section{Isolation and size separation of adipocyte}

Adipocytes were isolated according to a previously published procedure $^{(30)}$, with slight modifications. Briefly, freshly dissected epididymal adipose tissue (10g) from 16-week-old $\mathrm{fa} / \mathrm{fa}$ Zucker rats ( $n$ 5) was rinsed with PBS, minced with scissors and then incubated in minimum essential medium containing $1.05 \mathrm{mg} / \mathrm{ml}$ collagenase type A, 4\% bovine albumin and $1: 100 \mathrm{ml}$ antibiotic-antimycotic solution for $60 \mathrm{~min}$ at $37^{\circ} \mathrm{C}$. The digested samples were then filtered through a $250-\mu \mathrm{m}$ nitex screen (Dynamic Aqua-Supply, Surrey, BC, Canada) and washed three times with fresh media. The flow-through was collected in $50 \mathrm{ml}$ conical tubes that were gently inverted three times by hand, and the cells that resurfaced were collected and subsequently filtered through a $70-\mu \mathrm{m}$ nitex screen. To collect large adipocytes, the mesh was inverted over a new conical tube and washed with fresh media to capture the large adipocytes. Dense cells that did not surface after $30 \mathrm{~s}$ were filtered through a $50-\mu \mathrm{m}$ nitex screen and the cells that passed through were considered to be small adipocytes. Both small and large adipocyte populations were cultured and treated with CLA isomers, as described in the figure legend.

\section{Biochemical assays}

Adiponectin concentrations in the cell media were determined using an ELISA kit for total mouse/rat adiponectin (Otsuka Pharmaceutical, Montreal, QC, Canada). AngII concentrations were analysed using an AngII EIA kit (SPIbio, Montigny le 
Bretonneux, France) that has $4 \%$ cross-reactivity with angiotensin I and 36\% with angiotensin III. Absorbance readings for both assays were obtained with a microplate reader (ThermoMAX, Molecular Devices, Sunnyvale, CA, USA) using SOFTmax Pro software (Molecular Devices).

\section{Western blot analysis}

Cell lysates were analysed by Western blotting, as previously described $^{(31)}$ for angiotensinogen (1:1000; Fitzgerald, Concord, MA, USA) and adiponectin (1:1000; Calbiochem, Nottingham, UK). Membranes were stained with Ponceau S (Sigma, Oakville, ON, Canada) to confirm equal protein loading ${ }^{(31)}$. Data are expressed as arbitrary units after normalising to the loading control (Ponceau S).

\section{Statistical analysis}

Data were analysed with SAS 6.04 (SAS Institute, Inc., Cary,

to determine the effect of diet on organ weights, cell size and cell number, whereas the effects of diet and duration on

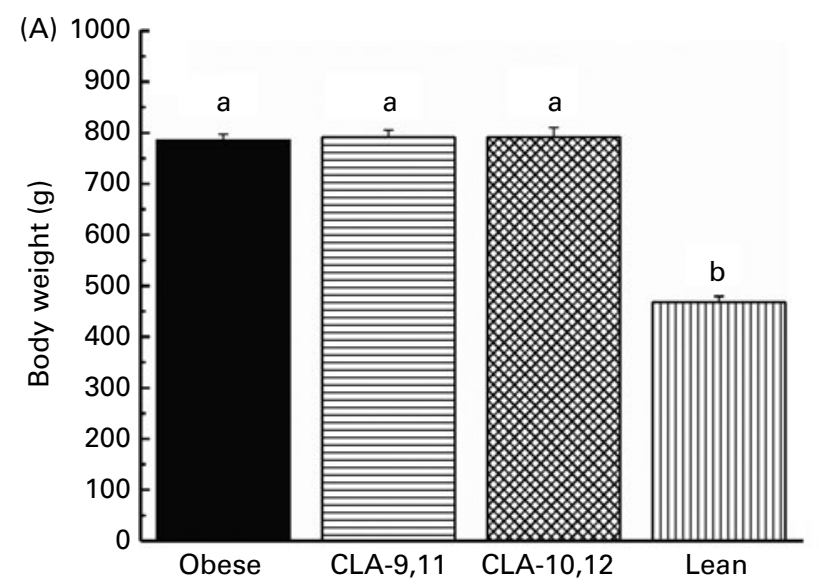

blood pressure were analysed as a two-way ANOVA, with main effects of time and diet. Students $t$ test was used to determine changes within a group over time, week 0 compared to week 8 . In the primary adipocyte experiments, differences in size between the two cell populations were analysed by Student's $t$ test, and a two-way ANOVA was used to determine the effect of cell size and treatment on adipokine production and secretion. Following ANOVA, differences among groups were determined by Duncan's multiple range test. A $P$ value of $\leq 0.05$ was considered to be statistically significant and all results are reported as mean values with their standard errors.

\section{Results}

\section{Body mass and blood pressure}

At the end of the 8-week feeding period, the food intake (data not shown), the body weight (Fig. 1(A)) and adipose tissue weight (Fig. 1(B)) of the obese $f a / f a$ Zucker rats were similar among the three groups, but were higher than that of the lean animals. In parallel with weight, all obese animals had a systolic pressure at baseline, almost $30 \mathrm{mmHg}$
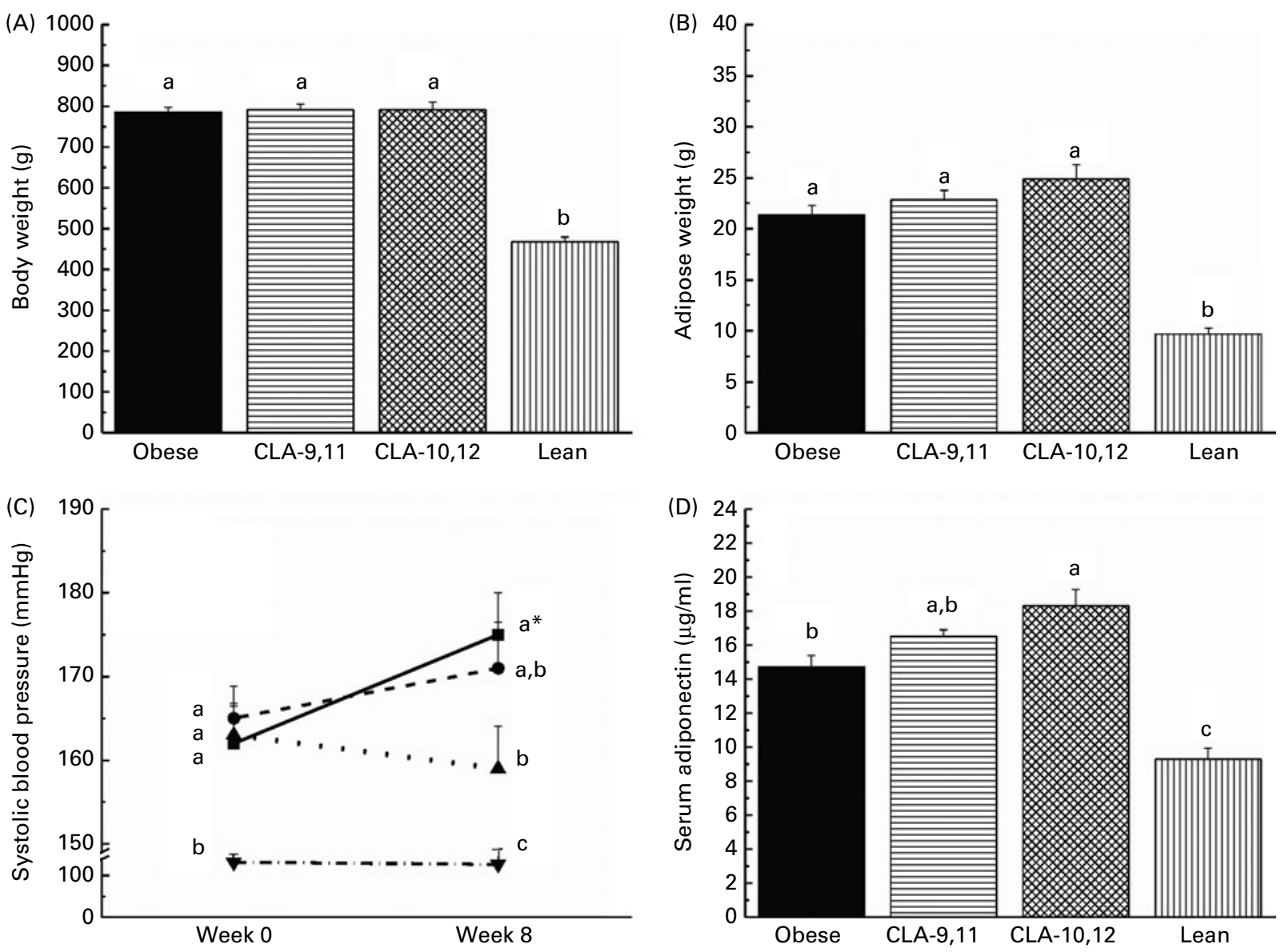

Fig. 1. Physiological parameters of fa/fa Zucker rats fed with conjugated linoleic acid (CLA) isomers for 8 weeks ( $n 5-10$ per group). (A) Body weight, (B) epididymal adipose tissue weight, $(C)$ systolic blood pressure and $(D)$ total serum adiponectin. Values are means with their standard errors represented by vertical bars. ${ }^{a, b, c}$ Mean values with unlike letters were significantly different between groups at the same time point $\left(P \leq 0 \cdot 05\right.$, Duncan's multiple range test). ${ }^{\star}$ Mean values were significantly different within a treatment group from week 0 to week $8(P \leq 0.05$, Student's $t$ test). Obese ( $\rightarrow-)$, fa/fa Zucker rats fed with $0 \%$ (w/w) CLA;

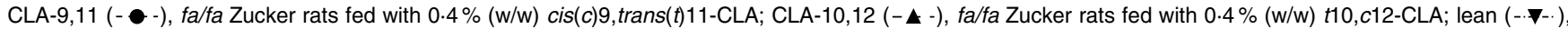
lean Zucker rats fed with $0 \%(w / w)$ CLA. 
higher than the lean (obese, 162 (sEm 4) mmHg; lean, 132 (SEM 3) $\mathrm{mmHg}$ ). Furthermore, unlike the lean animals in which blood pressure remained constant, systolic pressure increased in the $\mathrm{fa} / \mathrm{fa}$ control group by more than $10 \mathrm{mmHg}$ over the 8-week period of the present study (Fig. 1(C)), resulting in a $40 \mathrm{mmHg}$ difference with lean group (obese, 175 (sEM 5) mmHg; lean, 127 (SEM 2) mmHg). In contrast, supplementation with the $t 10, c 12$-CLA isomer prevented the increase in systolic blood pressure observed in the obese group at week 8 (159 (SEM 5) v. 175 (SEM 5) $\mathrm{mmHg}$, respectively; Fig. 1(C)). Even though there was a $3 \%$ reduction in blood pressure in the $t 10, c 12$-CLA group, the values still remained significantly higher than those of the lean animals (159 (SEm 5) v. 127 (SEM 2) $\mathrm{mmHg}$, respectively; Fig. 1(C)). At week 8, blood pressure values for the $c 9, t 11$-CLA were not different from either the control or $t 10, c 12$-CLA groups, indicating that the $c 9, t 11$-CLA isomer was not as effective as the $t 10, c 12$-CLA isomer for attenuation of blood pressure. Serum adiponectin was significantly higher in the $t 10, c 12$-CLA group (18.3 (SEM $1.0) \mu \mathrm{g} / \mathrm{ml})$ compared to the obese and lean groups $(15.7$ (SEM 0.7) and $9.3(\operatorname{sem~0.7)~} \mu \mathrm{g} / \mathrm{ml}$, respectively; Fig. 1(D)).

\section{Adipocyte size}

The obese control group had significantly larger cells in comparison to the lean control group (Fig. 2(A)). Adipocyte area was similar in both the group receiving the $c 9, t 11$-CLA isomer and the obese control group (Fig. 2(A)). In contrast, the adipocytes of the group receiving the $t 10, c 12$-CLA isomer were similar in size to those of the lean group. The adipocytes of these two groups ( $t 10, c 12$-CLA and lean) were $50 \%$ smaller than that in the obese control and $c 9, t 11$-CLA groups (Fig. 2(A)). The distribution pattern of adipocyte size shows that lean rats and obese rats receiving the $t 10, c 12$-CLA

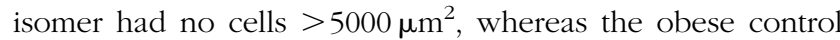
group and the obese rats receiving the $c 9, t 11$-CLA isomer had some cells in excess of $6000 \mu \mathrm{m}^{2}$ (Fig. 2(B)). The $t 10, c 12$-CLA group had 44 and $40 \%$ more adipocytes compared to the control and $c 9, t 11$-CLA groups, respectively, and a similar number of adipocytes compared to the lean group (Fig. 2(C)). It appears that the adipocytes from animals receiving the $t 10, c 12$ CLA isomer may function more like adipocytes from non-obese animals.

\section{Adipokine production by size-separated adipocytes}

To test whether the effects of CLA were direct, adipocytes isolated from epididymal adipose tissue of $f a / f a$ Zucker rats were size-separated and then treated with CLA isomers. The technique employed to separate adipocytes according to size achieved a 10-fold difference in the mean cell area between the large and small populations (Fig. 3(A) and (B)). This significant distinction in cell size enabled us to investigate the specific cytokines secreted by each cell population within the context of blood pressure regulation. Small adipocytes expressed significantly higher levels of adiponectin than large adipocytes (Fig. 4(A)). In contrast, small adipocytes had significantly lower levels of angiotensinogen than large
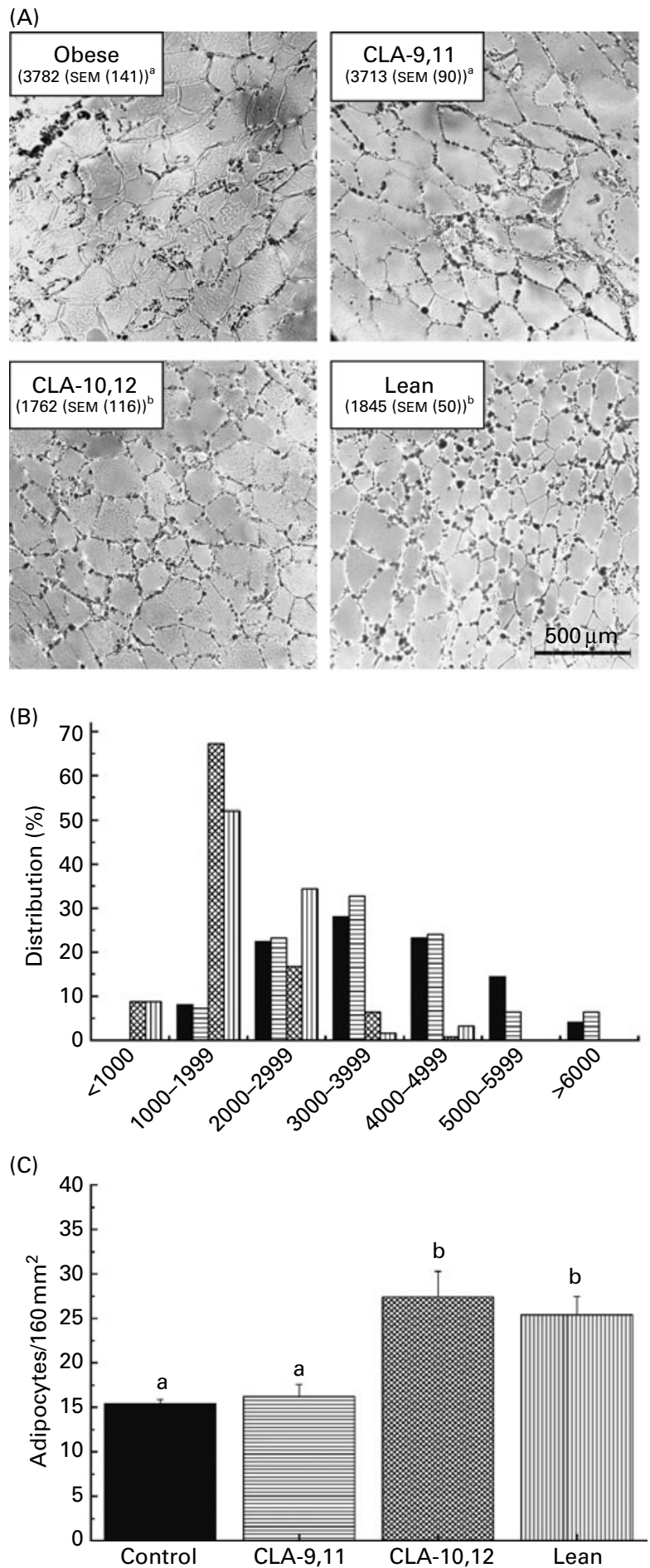

Fig. 2. Histology of epididymal adipose tissue from fa/fa Zucker rats fed with conjugated linoleic acid (CLA) isomers for 8 weeks ( $n 5$ per group). (A) Adipocyte size was quantified for each treatment group and cell area is reported in $\mu \mathrm{m}^{2}$ for the overall mean values with their standard errors ( $n 5$ per group). (B) The distribution of adipocyte size in each treatment group is also shown. (C) The number of adipocytes for each treatment group is reported as overall mean number of cells with their standard errors per $160 \mathrm{~mm}^{2}$. ${ }^{\mathrm{a}, \mathrm{b}}$ Mean values with unlike letters were significantly different between groups ( $P \leq 0.05$, Duncan's multiple range test). Obese $(\boldsymbol{a})$, fa/fa Zucker rats fed with $0 \%(\mathrm{w} / \mathrm{w})$ CLA; CLA-9,11 (目), fa/fa Zucker rats fed with $0.4 \%(\mathrm{w} / \mathrm{w})$ $\operatorname{cis}(c) 9, \operatorname{trans}(t) 11-\mathrm{CLA}$; CLA-10,12 (图), fa/fa Zucker rats fed with $0.4 \%$ (w/w)

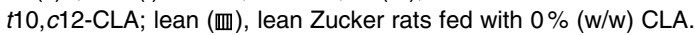


(A)

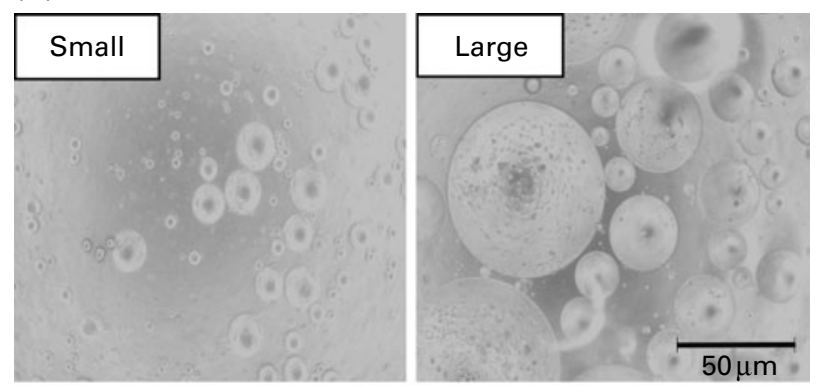

(B)

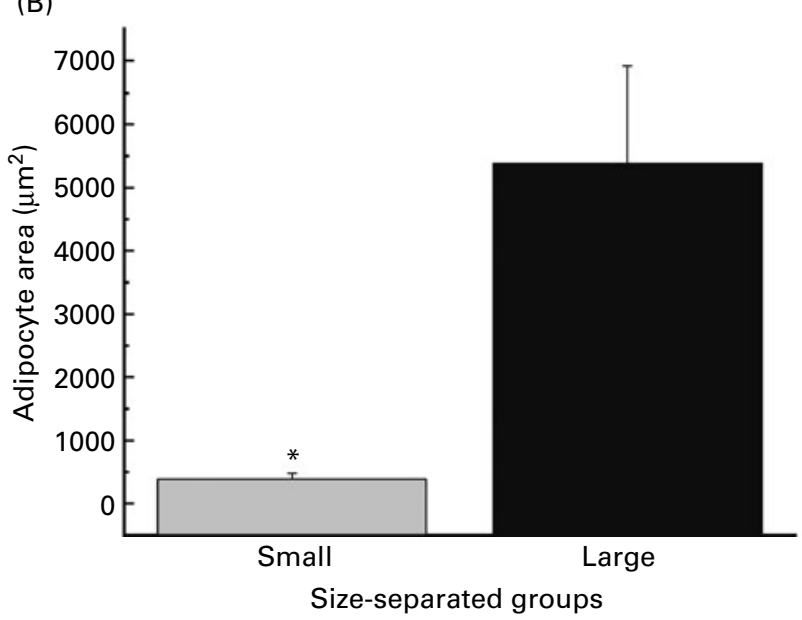

Fig. 3. Large and small adipocyte populations from fa/fa Zucker rats ( $n$ 5) (A) Representative images of small and large adipocytes obtained by size separation. (B) The area in $\mu \mathrm{m}^{2}$ of these cell populations was quantified. Values are means with their standard errors represented by vertical bars. * Mean values were significantly different between small adipocytes ( $P \leq 0.005$, Student's $t$ test)

adipocytes (Fig. 4(B)). CLA isomers did not significantly influence levels of adiponectin or angiotensinogen in large adipocytes (Fig. 4(A) and (B), respectively). However, in small adipocytes, both CLA isomers increased adiponectin levels (Fig. 4(A)). Differences in the adipokine content of the cell medium were also observed for the large and small adipocytes (Fig. 4(C) and (D)). Although treatment with the CLA isomers did not influence adiponectin secretion by either the small or large cells (Fig. 4(C)), the $c 9, t 11$-CLA isomer reduced AngII in the cell medium of large adipocytes compared to the linoleic acid control (Fig. 4(D)).

\section{Discussion}

The present investigation demonstrates for the first time that inclusion of the $t 10, c 12$-CLA isomer in the diet attenuates systolic blood pressure, increases serum adiponectin and leads to a decrease in adipocyte size in older $f a / f a$ Zucker rats that already have hypertension. Furthermore, adipocyte size reverts with $t 10, c 12$-CLA feeding to that of lean rats. These positive changes in blood pressure, adipocyte size and adiponectin occurred without a reduction in body weight, thus highlighting the importance of adipose tissue function. Lastly, synthesis and secretion of adipokines involved in blood pressure regulation are distinctly different in small and large adipoyctes, with higher levels of adiponectin in small adipocytes compared to large adipocytes, and the converse with angiotensinogen. The literature suggests that hypertrophic adipocytes have altered normal function, resulting in augmented production and secretion of adipokines that influence vascular function and contribute to obesity-related complications like hypertension ${ }^{(32-34)}$.

Hypertension exemplifies a condition for which there is excellent epidemiological evidence showing a positive relationship with obesity and which leads to serious health consequences $^{(35,36)}$. Owing to the increased incidence of obesity in our society, there is great demand for the development of novel lifestyle interventions and/or new pharmacological targets that can reduce the incidence of obesityrelated diseases. A reduction of as little as $9 \mathrm{mmHg}$ in systolic pressure significantly reduces the risk of a major vascular event or stroke in all BMI categories ${ }^{(37)}$. The present study was therefore designed to examine the utility of dietary intervention with CLA in the treatment of established obesityrelated hypertension. Although the $t 10, c 12$-CLA isomer only reduced systolic pressure by $3 \%$ over 8 weeks, at the end of the treatment period, there was a $17 \mathrm{mmHg}$ difference in systolic pressure between the control group and the group receiving the $t 10, c 12$-CLA isomer, a difference great enough to perhaps reduce the risk of a cardiovascular event.

We demonstrated that the $t 10, c 12$-CLA isomer is responsible for reducing epididymal adipocyte size and increases the number of adipoytes per unit area in older $f a / f a$ Zucker rats without decreasing body or adipose tissue weight. We have recently shown similar results in younger $f a / f a$ Zucker rats receiving the $t 10, c 12$-CLA isomer ${ }^{(5)}$. Most of the previous studies $^{(4,38,39)}$ investigating CLA and adipocyte size were conducted in growing animals using a mixture of CLA isomers. Data from these studies show an increase in the proportion of small adipocytes in growing female Sprague-Dawley or male $f a / f a$ Zucker rats fed with CLA mixtures, with soybean oil in the background diet. Interestingly, this effect is lost in male Wistar rats when saturated palm oil is used in the background diet ${ }^{(39)}$. In addition to our recent study in younger $\mathrm{fa} / \mathrm{fa}$ Zucker rats ${ }^{(5)}$, only a single study has looked at individual isomers of CLA and adipocyte size, and it showed that the $c 9, t 11$-CLA isomer increased adipocyte size in the inguinal and retroperitoneal depots of Wistar rats, but there was no effect when the isomers were combined ${ }^{(39)}$. These data suggest that the $t 10, c 12$-CLA isomer may be preventing the negative actions of the $c 9, t 11$-CLA isomer.

The reduction in adipocyte size observed with the $t 10, c 12$ CLA isomer suggests a possible improvement in adipocyte function and prompted the exploration of whether size is actually associated with improved cell function, particularly in the production and secretion of blood pressure-related adipokines. One of the adipokines that we focused on was angiotensinogen, because previous study showed that angiotensinogen-deficient mice have reduced adipocyte size as well as lower blood pressure compared to wild-type mice $^{(40)}$. Furthermore, overexpression of adipose tissue angiotensinogen results in increased adipocyte size and elevated 
(A)

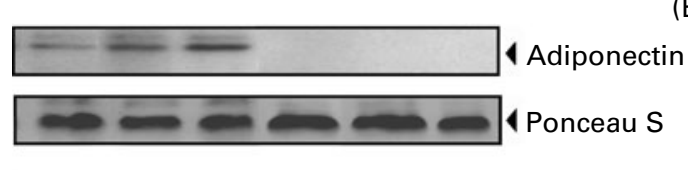

(B)

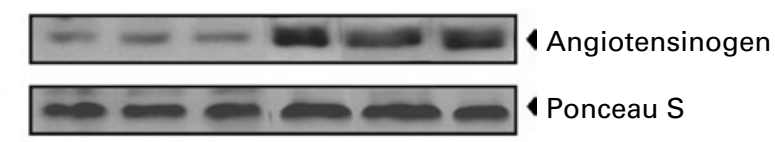

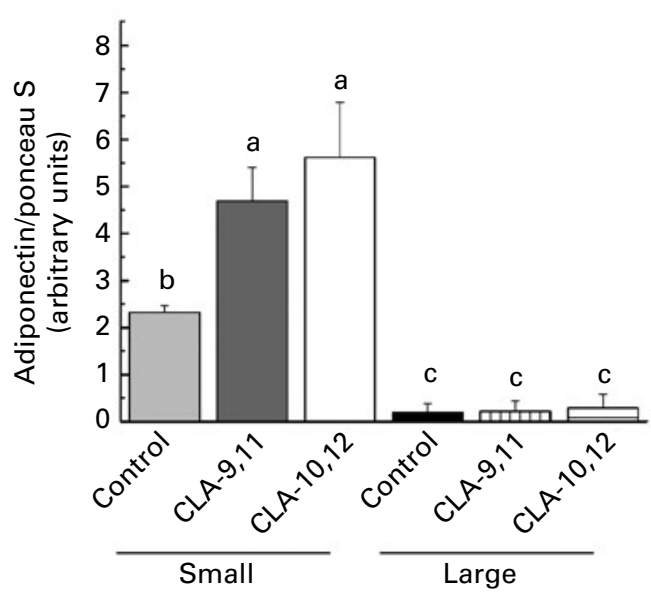

(C)

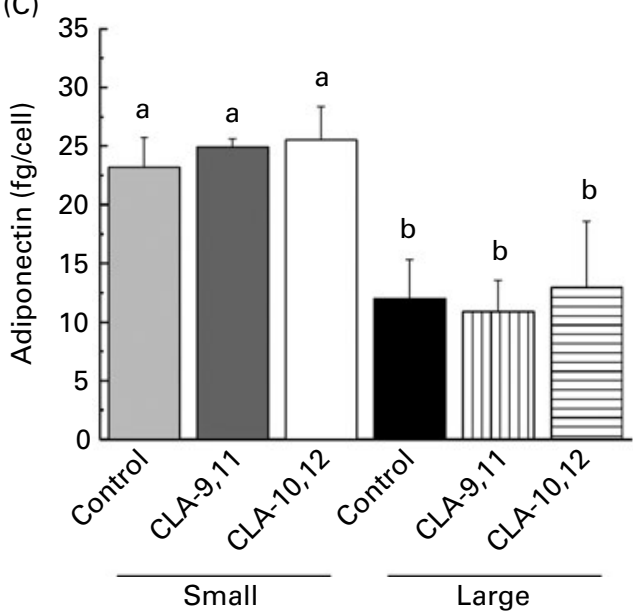

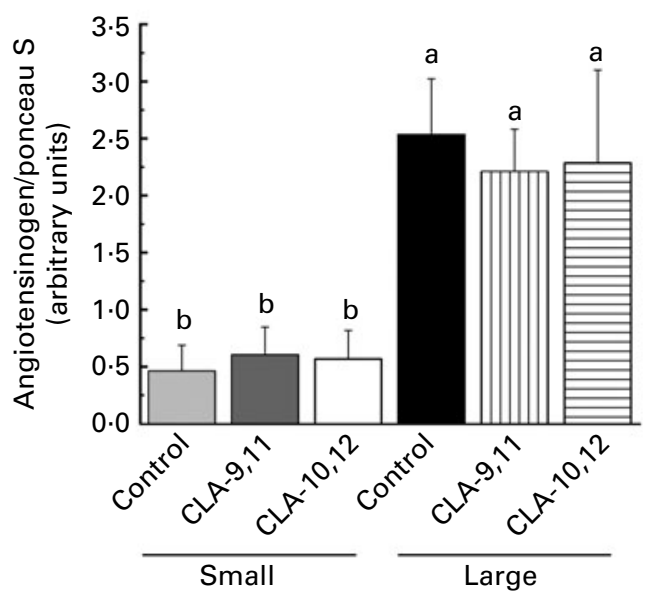

(D)

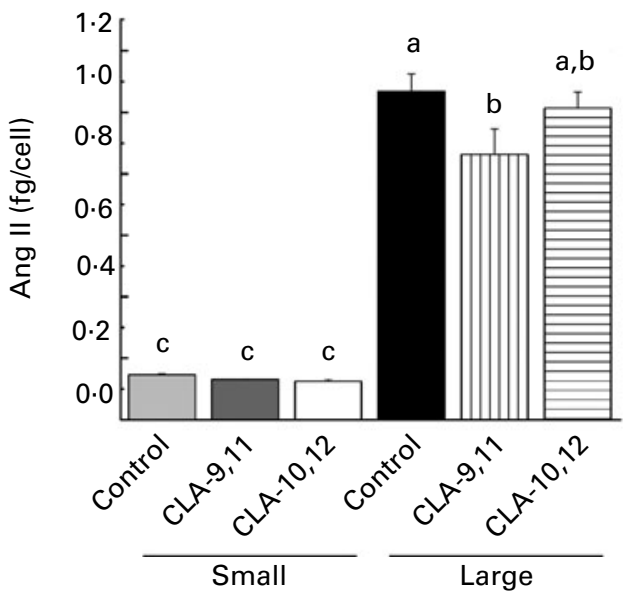

Fig. 4. Adipokines produced by large and small adipocytes from fa/fa Zucker rats $(n 5)$. Both small and large adipocytes were plated on six-well plates and treated

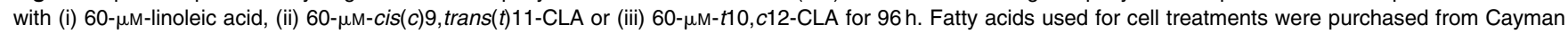
Chemical (Ann Arbor, MI, USA). Western blotting was subsequently used to analyse (A) adiponectin and (B) angiotensinogen levels in cells; representative blots are presented in the upper panel. Protein levels expressed as arbitrary units relative to Ponceau S (Sigma, Oakville, ON, Canada) staining for total protein. Enzyme immunoassays were used to analyse (C) total adiponectin and (D) angiotensin II (Ang II) in the culture media. Values are means with their standard errors represented by vertical bars. ${ }^{\text {a,b,c }}$ Mean values with unlike letters were significantly different between groups $(P \leq 0.05$, Duncan's multiple range test).

blood pressure ${ }^{(40)}$. In the present study, the use of size-separated adipocytes allowed us to study the treatment of different cell populations in vitro, and we observed differences in adipokine status from cells of the same animal. Large adipocytes expressed more angiotensinogen, resulting in higher amounts of AngII in the medium. These data add to those from a previous study by Hainault et $a l^{(41)}$, who reported that both secreted and cellular angiotensinogen levels are greater in obese compared to lean rat adipocytes. This model also allowed us to study the direct effects of CLA isomers on adipocytes of differing sizes. Although CLA isomers did not influence AngII secretion by small adipocytes, the $c 9, t 11$-CLA isomer reduced AngII secretion by large adipocytes. This may be a possible mechanism for the observed benefits noted in younger $f a / f a$ Zucker rats consuming a $c 9, t 11$-CLA isomer $\operatorname{diet}^{(42)}$. These results suggest that CLA isomers may independently contribute to the blood pressure effects of CLA.

Ohashi et $a l .{ }^{(16)}$ have shown that hypoadiponectinaemia is also associated with the development of obesity-related hypertension in a mouse model; however, until recently the mechanism of action was unclear. Intriguingly, weight loss of 5-10\% can increase circulating adiponectin concentrations and result in smaller adipocytes ${ }^{(43)}$. Likewise, the present study showed increased adiponectin expression and secretion in small adipocytes compared to large adipocytes. We also demonstrated that CLA isomers increase levels of adiponectin in small adipocytes from $\mathrm{fa} / \mathrm{fa}$ Zucker rats without affecting secretion of adiponectin. Therefore, intercellular signalling within adipose tissue must be responsible for regulating adiponectin release. This is not surprising, considering the previous study with adipose tissue in culture that showed no 
change in adiponectin mRNA with IL-6 treatment alone, although a decrease in adiponectin mRNA was observed when incubated with IL-6 as well as its soluble receptor ${ }^{(15)}$.

The ability of CLA to alter adiponectin production and secretion has largely been investigated with 3T3-L1 mouse adipocytes, which respond to the $c 9, t 11$-CLA isomer by increasing adipocyte adiponectin levels ${ }^{(44)}$ as well as adiponectin secretion $^{(45)}$, whereas the $t 10, c 12$-CLA-isomer decreases cellular and secreted levels of adiponectin ${ }^{(46)}$. In primary epididymal adipocytes from Wistar rats, a mixture of CLA isomers (up to $200 \mu \mathrm{M}$ ) decreased the secretion of $\operatorname{adiponectin}^{(47)}$. We did not note any differences in adiponectin secretion by CLA isomers in the present study; however, a comparison with other studies is difficult as cell size was not considered. The present study is unique in that it employs the use of size-separated adipocytes to understand the effects of CLA isomers in dysfunctional adipoyctes.

The differences in adiponectin levels in the size-separated adipocytes relate well with the in vivo data, which show that the reduced adipocyte size obtained by feeding the $t 10, c 12$-CLA isomer was associated with increased serum adiponectin concentrations in $f a / f a$ Zucker rats. Serum adiponectin concentrations were investigated in the present study, as previous research has demonstrated a negative correlation between blood pressure and serum adiponectin concentrations $^{(48)}$. Additionally, a recent study from our laboratory demonstrated a direct benefit on blood pressure by increasing adiponectin levels and activating endothelial nitric oxide synthase in $f a / f a Z^{2}$ ucker rats ${ }^{(42)}$, suggesting a mechanism for the actions of the $t 10, c 12$-CLA isomer. The present data show that obese $f a / f a$ Zucker rats have higher serum adiponectin concentrations than lean rats, unlike human subjects in whom adiponectin concentrations negatively correlated with obesity, and others have reported similar findings ${ }^{(49)}$. The higher levels of adiponectin in the obese rats compared to the lean rats may be due to a decrease in adiponectin receptor mRNA levels and increased secretion from brown adipose tissue $^{(49)}$. Even so, the present data are in agreement with previous studies showing that a mixture of CLA isomers increases serum adiponectin concentrations in disease models such as $\mathrm{fa} / \mathrm{fa}$ Zucker rats, Zucker diabetic fatty rats and spontaneously hypertensive rats ${ }^{(4,12,13)}$. These observations have recently been extended to human subjects by Zhao et al. ${ }^{(50)}$, who showed that the combination of CLA and ramipril led to a significant increase in circulating adiponectin levels in obese hypertensive human subjects.

Finally, the present study adds to current knowledge by demonstrating that the $t 10, c 12-C L A$ isomer provides beneficial effects on systolic blood pressure in an older animal model that already has hypertension, in contrast to previous reports that have shown that CLA isomers may be beneficial for preventing the development of hypertension in growing rats ${ }^{(11-13,42)}$. Interestingly, Zhao et al. ${ }^{(50)}$ recently published a study in obese hypertensive men and women, showing that CLA supplementation $(4.5 \mathrm{~g} / \mathrm{d})$ enhanced the blood pressure-lowering effect of ramipril, an angiotensinconverting enzyme inhibitor. As a mixture of CLA isomers $(5.5 \mathrm{~g} / \mathrm{d})$ can be safely consumed by healthy human males
(BMI $<30 \mathrm{~kg} / \mathrm{m}^{2}$ ) with normal blood pressure without causing hypotension ${ }^{(51)}$, the blood pressure-lowering effects of CLA appear to be therapeutic, producing desirable results in disease conditions such as obesity-related hypertension.

In conclusion, the present study is the first to show that the $t 10, c 12$-CLA isomer displays beneficial effects on systolic blood pressure in adult hypertensive $f a / f a$ Zucker rats. We also showed beneficial effects of the $t 10, c 12$-CLA isomer on adipocyte size, and distinct differences in cellular and secretion levels of adipokines involved in blood pressure regulation from small and large adipocytes. However, treatment of isolated adipocytes with individual CLA isomers did not mimic the effects on adipokines seen in vivo, thus suggesting that cross-talk between various cells types present in adipose tissue may have an important role in regulating the production and release of adipokines in vivo. Further research investigating individual as well as combined CLA isomers in different obese hypertensive models looking at both total and size-separated adipocytes is warranted to better understand the effects of CLA isomers on adipocyte function in the context of adipokine production and secretion. On the other hand, the present study clearly establishes the existence of a link between the ability of CLA to improve blood pressure, increase adiponectin and reduce adipocyte size; however, the causal relationships among these benefits require further exploration.

\section{Acknowledgements}

The authors thank Crystal Bernier and Lisa Rigaux for their help with the animal care. Financial support was provided by the Dairy Farmers of Canada (C. G. T. and P. Z.), the Canada-Manitoba Agrifood Research Development Initiative (C. G. T. and P. Z.) and the National Science and Engineering Council Postgraduate Scholarship (V. D.), as well as the St Boniface Hospital and Research Foundation towards infrastructure support. V. D., C. G. T. and P. Z. envisioned and designed the experiments. V. D. preformed the experiments and analysed the data. V. D. wrote the draft of the manuscript, C. G. T. and P. Z. critically reviewed and revised the manuscript. All authors reviewed and approved the final version of the manuscript. The authors declare no conflicts of interest.

\section{References}

1. Trayhurn P (2007) Adipocyte biology. Obes Rev 8, Suppl. 1, 41-44.

2. Hajer GR, van Haeften TW \& Visseren FL (2008) Adipose tissue dysfunction in obesity, diabetes, and vascular diseases. Eur Heart J 29, 2959-2971.

3. Jo J, Gavrilova O, Pack S, et al. (2009) Hypertrophy and/or hyperplasia: dynamics of adipose tissue growth. PLoS Comput Biol 5, e1000324.

4. Noto A, Zahradka P, Yurkova N, et al. (2007) Dietary conjugated linoleic acid decreases adipocyte size and favorably modifies adipokine status and insulin sensitivity in obese, insulin-resistant rats. Metabolism 56, 1601-1611.

5. DeClercq V, Zahradka P \& Taylor CG (2010) Dietary $t 10, c 12$-CLA but not $c 9, t 11$ CLA reduces adipocyte size in 
the absence of changes in the adipose renin-angiotensin system in fa/fa Zucker rats. Lipids 45, 1025-1033.

6. Skurk T, Alberti-Huber C, Herder C, et al. (2007) Relationship between adipocyte size and adipokine expression and secretion. J Clin Endocrinol Metab 92, 1023-1033.

7. DeClercq V, Taylor C \& Zahradka P (2008) Adipose tissue: the link between obesity and cardiovascular disease. Cardiovasc Hematol Disord Drug Targets 8, 228-237.

8. Pausova $Z$ (2006) From big fat cells to high blood pressure: a pathway to obesity-associated hypertension. Curr Opin Nephrol Hypertens 15, 173-178.

9. Boustany CM, Bharadwaj K, Daugherty A, et al. (2004) Activation of the systemic and adipose renin-angiotensin system in rats with diet-induced obesity and hypertension. Am J Physiol Regul Integr Comp Physiol 287, R943-R949.

10. Engeli S, Negrel R \& Sharma AM (2000) Physiology and pathophysiology of the adipose tissue renin-angiotensin system. Hypertension 35, 1270-1277.

11. Nagao K, Inoue N, Wang YM, et al. (2003) The 10trans,12cis isomer of conjugated linoleic acid suppresses the development of hypertension in Otsuka Long-Evans Tokushima fatty rats. Biochem Biophys Res Commun 306, 134-138.

12. Nagao K, Inoue N, Wang YM, et al. (2003) Conjugated linoleic acid enhances plasma adiponectin level and alleviates hyperinsulinemia and hypertension in Zucker diabetic fatty ( $\mathrm{fa} / \mathrm{fa}$ ) rats. Biochem Biophys Res Commun 310, 562-566.

13. Inoue N, Nagao K, Hirata J, et al. (2004) Conjugated linoleic acid prevents the development of essential hypertension in spontaneously hypertensive rats. Biochem Biophys Res Commun 323, 679-684.

14. Arita Y, Kihara S, Ouchi N, et al. (1999) Paradoxical decrease of an adipose-specific protein, adiponectin, in obesity. Biochem Biophys Res Commun 257, 79-83.

15. Bruun JM, Lihn AS, Verdich C, et al. (2003) Regulation of adiponectin by adipose tissue-derived cytokines: in vivo and in vitro investigations in humans. Am $J$ Physiol Endocrinol Metab 285, E527-E533.

16. Ohashi K, Kihara S, Ouchi N, et al. (2006) Adiponectin replenishment ameliorates obesity-related hypertension. Hypertension 47, 1108-1116.

17. Wang Y, Xu A, Knight C, et al. (2002) Hydroxylation and glycosylation of the four conserved lysine residues in the collagenous domain of adiponectin. Potential role in the modulation of its insulin-sensitizing activity. $\mathrm{J} \mathrm{Biol} \mathrm{Chem}$ 277, 19521-19529.

18. Wang Y, Lam KS, Chan L, et al. (2006) Post-translational modifications of the four conserved lysine residues within the collagenous domain of adiponectin are required for the formation of its high molecular weight oligomeric complex. J Biol Chem 281, 16391-16400.

19. $\mathrm{Xu}$ A, Chan KW, Hoo RL, et al. (2005) Testosterone selectively reduces the high molecular weight form of adiponectin by inhibiting its secretion from adipocytes. $J$ Biol Chem 280, 18073-18080.

20. Kadowaki T, Yamauchi T, Kubota N, et al. (2006) Adiponectin and adiponectin receptors in insulin resistance, diabetes, and the metabolic syndrome. J Clin Invest 116, 1784-1792.

21. Yiannikouris F, Gupte M, Putnam K, et al. (2010) Adipokines and blood pressure control. Curr Opin Nephrol Hypertens 19, 195-200.

22. Thatcher S, Yiannikouris F, Gupte M, et al. (2009) The adipose renin-angiotensin system: role in cardiovascular disease. Mol Cell Endocrinol 302, 111-117.

23. Boustany CM, Bharadwaj K, Daugherty A, et al. (2004) Activation of the systemic and adipose renin-angiotensin system in rats with diet-induced obesity and hypertension. Am J Physiol Regul Integr Comp Physiol 287, R943-R949.

24. Ouchi N, Ohishi M, Kihara S, et al. (2003) Association of hypoadiponectinemia with impaired vasoreactivity. Hypertension 42, 231-234.

25. Dunbar JC, Hu Y \& Lu H (1997) Intracerebroventricular leptin increases lumbar and renal sympathetic nerve activity and blood pressure in normal rats. Diabetes 46, 2040-2043.

26. Rahmouni K \& Morgan DA (2007) Hypothalamic arcuate nucleus mediates the sympathetic and arterial pressure responses to leptin. Hypertension 49, 647-652.

27. Ruth MR, Taylor CG, Zahradka P, et al. (2008) Abnormal immune responses in $f a / f a$ Zucker rats and effects of feeding conjugated linoleic acid. Obesity (Silver Spring) 16, $1770-1779$.

28. Feng M, Whitesall S, Zhang Y, et al. (2008) Validation of volume-pressure recording tail-cuff blood pressure measurements. Am J Hypertens 21, 1288-1291.

29. Whitesall SE, Hoff JB, Vollmer AP, et al. (2004) Comparison of simultaneous measurement of mouse systolic arterial blood pressure by radiotelemetry and tail-cuff methods. Am J Physiol Heart Circ Physiol 286, H2408-H2415.

30. Jernas M, Palming J, Sjoholm K, et al. (2006) Separation of human adipocytes by size: hypertrophic fat cells display distinct gene expression. FASEB J 20, 1540-1542.

31. Yau L, Litchie B, Thomas S, et al. (2003) Endogenous monoADP-ribosylation mediates smooth muscle cell proliferation and migration via protein kinase $\mathrm{N}$-dependent induction of c-fos expression. Eur J Biochem 270, 101-110.

32. Ahima RS \& Flier JS (2000) Adipose tissue as an endocrine organ. Trends Endocrinol Metab 11, 327-332.

33. Ahima RS (2006) Adipose tissue as an endocrine organ. Obesity (Silver Spring) 14, Suppl. 5, 242S-249S.

34. DeClercq V, Taylor C \& Zahradka P (2008) Adipose tissue: the link between obesity and cardiovascular disease. Cardiovasc Hematol Disord Drug Targets 8, 228-237.

35. Rexrode KM, Buring JE \& Manson JE (2001) Abdominal and total adiposity and risk of coronary heart disease in men. Int J Obes Relat Metab Disord 25, 1047-1056.

36. Rexrode KM, Carey VJ, Hennekens CH, et al. (1998) Abdominal adiposity and coronary heart disease in women. JAMA 280, 1843-1848.

37. Czernichow S, Ninomiya T, Huxley R, et al. (2010) Impact of blood pressure lowering on cardiovascular outcomes in normal weight, overweight, and obese individuals: the Perindopril Protection Against Recurrent Stroke Study trial. Hypertension 55, 1193-1198.

38. Azain MJ, Hausman DB, Sisk MB, et al. (2000) Dietary conjugated linoleic acid reduces rat adipose tissue cell size rather than cell number. $J$ Nutr 130, 1548-1554.

39. Lopes PA, Martins SV, Pinho MS, et al. (2008) Diet supplementation with the cis-9,trans-11 conjugated linoleic acid isomer affects the size of adipocytes in Wistar rats. Nutr Res 28, 480-486.

40. Massiera F, Bloch-Faure M, Ceiler D, et al. (2001) Adipose angiotensinogen is involved in adipose tissue growth and blood pressure regulation. FASEB J 15, 2727-2729.

41. Hainault I, Nebout G, Turban S, et al. (2002) Adipose tissuespecific increase in angiotensinogen expression and secretion in the obese $(f a / f a)$ Zucker rat. Am J Physiol Endocrinol Metab 282, E59-E66.

42. DeClercq V, Taylor CG, Wigle J, et al. (2011) Conjugated linoleic acid improves blood pressure by increasing adiponectin and endothelial nitric oxide synthase activity. J Nutr Biochem (epublication ahead of print version 16 June 2011). 
43. Varady KA, Tussing L, Bhutani S, et al. (2009) Degree of weight loss required to improve adipokine concentrations and decrease fat cell size in severely obese women. Metabolism 58, 1096-1101.

44. Joseph SV, Miller JR, McLeod RS, et al. (2009) Effect of trans 8 , cis $10+$ cis 9 , trans 11 conjugated linoleic acid mixture on lipid metabolism in 3T3-L1 cells. Lipids 44, 613-620.

45. Ahn IS, Choi BH, Ha JH, et al. (2006) Isomer-specific effect of conjugated linoleic acid on inflammatory adipokines associated with fat accumulation in 3T3-L1 adipocytes. $\mathrm{J} \mathrm{Med} \mathrm{Food}$ 9, 307-312.

46. Miller JR, Siripurkpong P, Hawes J, et al. (2008) The trans-10, cis-12 isomer of conjugated linoleic acid decreases adiponectin assembly by PPARgamma-dependent and PPARgammaindependent mechanisms. J Lipid Res 49, 550-562.

47. Perez-Matute P, Marti A, Martinez JA, et al. (2007) Conjugated linoleic acid inhibits glucose metabolism, leptin and adiponectin secretion in primary cultured rat adipocytes. Mol Cell Endocrinol 268, 50-58.

48. Chow WS, Cheung BM, Tso AW, et al. (2007) Hypoadiponectinemia as a predictor for the development of hypertension: a 5-year prospective study. Hypertension 49 , 1455-1461.

49. Oana F, Takeda H, Hayakawa K, et al. (2005) Physiological difference between obese $(f a / f a)$ Zucker rats and lean Zucker rats concerning adiponectin. Metabolism 54, 995-1001.

50. Zhao WS, Zhai JJ, Wang YH, et al. (2009) Conjugated linoleic acid supplementation enhances antihypertensive effect of ramipril in Chinese patients with obesity-related hypertension. Am J Hypertens 22, 680-686.

51. Raff M, Tholstrup T, Sejrsen K, et al. (2006) Diets rich in conjugated linoleic acid and vaccenic acid have no effect on blood pressure and isobaric arterial elasticity in healthy young men. J Nutr 136, 992-997. 